\title{
Economía de la arqueología: valor e impacto económico del patrimonio arqueológico
}

\section{Economics of archeology: value and economic impact of archaeological heritage}

\author{
Luis César Herrero Prieto \\ Universidad de Valladolid
}

\begin{abstract}
Resumen
El objetivo de este ensayo es presentar las bases analíticas de la economía de la arqueología. Se caracteriza el patrimonio arqueológico como un bien económico de características particulares, como su carácter dotacional e irreproducible, sometido a condiciones de sostenibilidad, que reúne las peculiaridades de un bien público o recurso de propiedad común, vinculado además a escenarios de información imperfecta. Estas características hacen que las condiciones de provisión no estén bien resueltas en una economía de mercado. Se profundiza, entonces, en los componentes de valor de la arqueología, distinguiendo entre valor cultural, valor económico, valor social e impacto económico. Se muestran finalmente distintas metodologías y aplicaciones sobre valoración e impacto económico del patrimonio arqueológico.
\end{abstract}

Palabras clave: economía de la cultura, economía de la arqueología, valoración del patrimonio histórico.

Clasificación JEL: Z10, Z11.

\begin{abstract}
The aim of this essay is to present the analytical bases of the economics of archaeology. Archaeological heritage can be defined as an economic good which displays particular features such as its endowment and irreproducible nature, added to which it is subject to conditions of sustainability. It also evidences the peculiarities of a public good or even common good, which is also linked to conditions of uncertainty. Such characteristics mean that the circumstances under which it is supplied are not well defined in a market economy. The value components of archaeology are thus explored in depth, distinguishing between cultural value, economic value, social value and economic impact. Finally, various valuation and economic impact techniques and applications of archaeology heritage are shown.
\end{abstract}

Keywords: cultural economics, economics of archeology, economic valuation of cultural heritage.

JEL classification: Z10, Z11. 


\section{Introducción}

Está en la naturaleza humana el afán de curiosidad por el conocimiento y el uso de la imaginación para la creación y la distracción. El patrimonio arqueológico combina ambos empeños ya que, por un lado, constituye la expresión de un esfuerzo intelectual por descubrir y razonar qué sucedió en el pasado, desde nuestros ancestros más remotos hace miles de años, hasta nuestro pasado reciente; y, por otro, responde a la necesidad más íntima de imaginar que hubo otro tiempo para cualquier lugar y de entender qué hemos sido y cómo hemos vivido. Se trata, por tanto, de un anhelo por dar continuidad a la memoria, de manera de enriquecer también el tiempo en que vivimos. Un mundo sin memoria es un mundo depravado, inexplicable. Pero, a la vez, el conocimiento del pasado y sus evidencias, son una fuente para nutrir la curiosidad, la distracción y la identidad (Demas y Agnew, 2013).

De este modo, la arqueología, entendida como ciencia que estudia las artes, los monumentos y los objetos de la antigüedad, especialmente a través de los restos encontrados (DRAE, 23.a ed., 2014), entronca con estas constancias del ser humano, y ha dado lugar a una de las disciplinas más nítidas y más clásicas en el ámbito de los estudios de historia y las humanidades. La actividad arqueológica ha girado tradicionalmente alrededor de la realización de excavaciones, que siempre han sido la parte central de la disciplina, y los métodos científicos de interpretación del material arqueológico, aun cuando ocupa un papel cada vez más importante la discusión sobre los criterios de conservación, en respuesta a los distintos modelos de explotación y valoración de los yacimientos arqueológicos que se han sucedido a lo largo de la historia de esta disciplina (Pérez-Juez, 2006). En esta labor, que en definitiva versa sobre la manera de proporcionar utilidad social al legado arqueológico, cobran importancia también las funciones de gestión de los recursos arqueológicos y la estrategia de exhibición y educación.

Sobre estas premisas nos preguntamos, entonces, por cuál es el papel de la economía en este contexto, qué puede aportar el análisis económico en la explicación de una actividad tan específica, los recursos que se derivan y los esfuerzos que se dedican. Algunos piensan todavía que economía y cultura, en general, son dos ámbitos incompatibles, en el sentido de que los bienes culturales agotan su razón de ser cuando pasan a los circuitos de producción y consumo, como si la economía degradase el valor intelectual y estético de la creación artística o el patrimonio cultural. Es más, a veces se reprocha que algunos males asociados a la congestión turística o la perversión del significado cultural son consecuencia precisamente de una excesiva mercantilización de la cultura y la obsesión por la búsqueda de rentabilidad. Sin embargo, nuestro planteamiento parte de un dilema básico y típico del análisis económico: la arqueología es una actividad consumidora de recursos, fundamentalmente por una vocación intelectual como es el estudio de las sociedades antiguas a través de sus restos materiales, para dar cumplimiento a determinadas necesidades, algunas de orden científico y propósitos de preservación, que suelen estar vinculadas a una determinada elección social, y otras de orden privado asociadas al interés por el 
patrimonio arqueológico y las distintas modalidades de participación y consumo cultural. Este dilema es de naturaleza económica y, por tanto, preguntarnos por el coste de oportunidad de un proyecto arqueológico, por el valor del legado patrimonial o el impacto de una intervención, no son un tipo de extravagancia ni una provocación, sino cuestiones que atañen a la economía y que la economía puede tratar de resolver.

En consecuencia, nuestro propósito en este ensayo es presentar la capacidad explicativa del análisis económico en el problema de asignación de recursos de esta actividad, y esbozar lo que serían las bases analíticas de la economía de la arqueología. Desde nuestro conocimiento, no existen muchos trabajos que traten de caracterizar la arqueología desde el punto de vista económico. Pueden mencionarse como primeros esfuerzos el trabajo de Weigel (2006) y las aportaciones al número monográfico de la revista Public Arquaeology sobre arqueología y desarrollo económico editado por Gould y Burtehnshaw (2014). No obstante, los yacimientos arqueológicos han sido objeto de estudio relativamente frecuente en ejercicios de valoración económica e impacto turístico, como más adelante veremos. De este modo, hacemos frente a este desafío analítico con este ensayo que está dividido en cinco secciones. Después de esta introducción, la segunda sección aborda algunas transformaciones recientes en la arqueología, que dan lugar a determinados terrenos comunes con la economía, y se aporta una definición del ámbito analítico de la arqueología desde el punto de vista del análisis económico. La sección tercera se orienta a la caracterización del patrimonio arqueológico como un bien económico de características particulares. La sección cuarta aborda los distintos componentes de valor de la arqueología y se muestran distintas metodologías y aplicaciones sobre valor e impacto económico del patrimonio arqueológico. El trabajo termina con una síntesis de conclusiones.

\section{Economía y arqueología: territorios comunes}

Quizás pudiéramos pensar que la arqueología debiera ser una actividad minoritaria por su especificidad. Sin embargo, resulta curioso comprobar cómo en la realidad el ámbito de esta profesión se ha extendido de manera significativa, y es muy frecuente encontrar arqueólogos en funciones de gestión cultural y en la dirección de proyectos y políticas culturales en vez de en excavaciones arqueológicas. No es nuestro propósito en este momento delimitar afinidades y campos competenciales, sino avanzar en la acepción de arqueología para un economista y constatar que, como cualquier otra actividad económica, se trata de una tarea que consume recursos de acuerdo a determinadas finalidades o preferencias, sobre las que cabe valorar su asignación óptima. La posibilidad de aporte explicativo por parte de la economía está, por tanto, en la propia naturaleza de la actividad. Pero, además, de manera complementaria a esta afirmación, se comprueba que en la arqueología se están produciendo algunas transformaciones importantes, interesantes también para el análisis económico, que merece señalar. 
En primer lugar, se ha producido una extensión del concepto de patrimonio arqueológico, que ya no se ciñe exclusivamente a las civilizaciones antiguas, sino que se amplía al análisis de restos materiales cada vez más recientes. Véase en este sentido el alcance de la denominada arqueología industrial, o la consideración del patrimonio tecnológico asociado a oficios, profesiones y actividades económicas y que se centran, por tanto, en el rescate y estudio de legados patrimoniales fundamentalmente de los siglos XIX y xx (Buchanan, 2005). También existe una extensión hacia la consideración de elementos intangibles como el patrimonio cultural inmaterial y también el patrimonio del paisaje natural humanizado, que incluso han dado lugar a nuevas Cartas de la UNESCO y denominaciones en la clasificación de Patrimonio de la Humanidad (Vecco, 2010). Por tanto, se ha producido una diversificación del producto arqueológico, es decir, una ampliación de la oferta de patrimonio cultural asociado a esta disciplina.

En segundo lugar, la arqueología no es ya una actividad solo anclada en técnicas trabajo-intensivas, como las tradicionales excavaciones en tierra, sino que admite aportes de capital productivo y es permeable al cambio técnico y las innovaciones. Pueden comprobarse, en este sentido, desde los progresos recabados en los problemas de datación y análisis de restos arqueológicos, hasta los resultados de la arqueología aérea, arqueología subacuática, etc. (Fernández Díaz, 2016) que han permitido incrementar la producción y la eficiencia en el trabajo arqueológico. En definitiva, la arqueología, como actividad vinculada al descubrimiento analítico, diversifica su función de producción con factores sustitutivos y pueden darse mejoras notables en productividad debido al progreso técnico.

Por último, la arqueología, hoy en día, no se circunscribe al rescate del legado patrimonial, sino que en múltiples ocasiones avanza un paso más en la confección de una oferta cultural a partir del recurso patrimonial originario. De este modo, el patrimonio arqueológico está recibiendo nuevos usos y dando lugar a nuevas dotaciones arquitectónicas, así como la expansión hacia una nueva oferta cultural y turística de bienes y servicios derivados (Paardekooper, 2012). Como ejemplos de esta tendencia pueden constatarse, por ejemplo, el aprovechamiento de algunos anfiteatros griegos y romanos para la celebración de festivales de artes escénicas y conciertos musicales ${ }^{1}$; la extensión de los denominados museos de sitio y centros de interpretación, con la construcción de nuevos edificios, algunos de excelente factura que se convierten en un atractivo complementario al yacimiento arqueológico ${ }^{2}$; o la inspiración que la arqueología provoca en algunas industrias culturales reproducibles, desde la joyería, la artesanía y la moda, hasta las industrias de contenido, como

${ }^{1}$ En España tenemos, desde uno de los festivales más antiguos y acreditados, como es el Festival de Teatro Clásico de Mérida, a los conciertos que ocasionalmente se organizan, por ejemplo, en el teatro romano de Clunia, en Burgos, y así otros muchos ejemplos.

${ }_{2}^{2}$ De nuevo puede considerarse el Museo Nacional de Arte Romano en Mérida, una de las obras más emblemáticas del arquitecto Rafael Moneo, pero también otras muchas instalaciones surgidas junto a yacimientos arqueológicos de relieve. Véanse como ejemplos la Villa Romana de la Olmeda en Palencia, o Almenara-Puras en Valladolid. 
vídeos, películas, etc. ${ }^{3}$. Por tanto, el patrimonio arqueológico no es tan solo un recurso inmanente, sino también origen de una pléyade de nuevos productos y servicios, que son demandados por la sociedad y dan lugar a consumos y mercados específicos y no solo de tipo turístico.

Sobre la base de estas comprobaciones, a mi juicio y en síntesis, la arqueología dispone de dos componentes estrechamente ligados y que se combinan en distintas proporciones en cada caso o cada prototipo arqueológico (Figura 1): por una parte, el conocimiento del pasado, basado en premisas estrictamente científicas, y por otra parte, la imaginación sobre el pasado, cuya evocación también es compartida e incluso provocada por los propios arqueólogos e investigadores ${ }^{4}$, aunque constituye la precepción básica del resto de los individuos cuando visitan y disfrutan del patrimonio arqueológico como turistas o curiosos, bien sea en los yacimientos, en los museos, o en cualquier otra forma de consumo de sus productos derivados (Ross et al., 2017). En esta combinación de intereses y de usos del patrimonio arqueológico, la perspectiva del análisis económico establece que la sociedad discrimina recursos para el logro de los primeros fines, es decir, los asociados a motivos de investigación y conocimiento, que suelen estar provistos de manera pública a instancias de una decisión colectiva; mientras que distribuye y jerarquiza la asignación de recursos de acuerdo a los consumos y preferencias respecto de los segundos, es decir, la imaginación asociada al ocio y disfrute del patrimonio arqueológico. El problema radica en que, mientras en estas segundas aplicaciones, en tanto que rentables y nítidas en su delimitación correcta en el modo de producción y apropiabilidad, se suelen resolver bien a través del mercado y de los precios, en las primeras funciones hay problemas de delimitación de la manera de apropiación de resultados, y la sociedad interviene en forma de decisiones colectivas fundamentadas en los criterios de bien de mérito o fallo de mercado. Este es el problema fundamental de la asignación de recursos en el ámbito de la arqueología, ya que la determinación del valor económico del legado arqueológico está fundamentada en una necesidad primordial de reconocimiento social de su valor cultural (Klamer, 2014) y, por tanto, el impacto económico se circunscribe ocasionalmente a los elementos más acreditados, mientras que los recursos arqueológicos menores quedan desamparados de reconocimiento de valor e impacto. Por eso resulta importante establecer bien la conceptualización del patrimonio arqueológico como bien económico y profundizar posteriormente en los problemas y prácticas de estimación de valor, cuestiones que se acometen seguidamente.

\footnotetext{
${ }^{3}$ En el ámbito de los productos audiovisuales, hablar del impacto de las películas de Indiana Jones o algo más cercano como la serie animada española tan premiada de Tadeo Jones, pueda parecer un exceso en justificar la arqueología como inspiración, pero no tanto si observamos el alcance y reconocimiento que han tenido, por ejemplo, las obras de Mary Beard sobre el mundo romano, tanto literarias como la serie de videos para la BBC. Por lo que se refiere a artefactos creativos, no es desdeñable como fuente de actividad la producción artesana que rodea los principales yacimientos arqueológicos, pero también las producciones más delicadas de las boutiques de los museos de sitio, o incluso y por ejemplo la creación de joyería de inspiración etrusca en Italia o vaccea y tartesia en España.

${ }^{4}$ Cabe preguntarse cuánto de idealización o rigor presentan la disposición de muchos conjuntos arqueológicos, que está en la base de una discusión muy prolija entre arqueólogos y arquitectos sobre los criterios de conservación purista o idealizada, y que se suelen asociar con las posiciones contrapuestas de Ruskin y Viollet-le-Duc, respectivamente. Vid. Pérez-Juez (2006).
} 


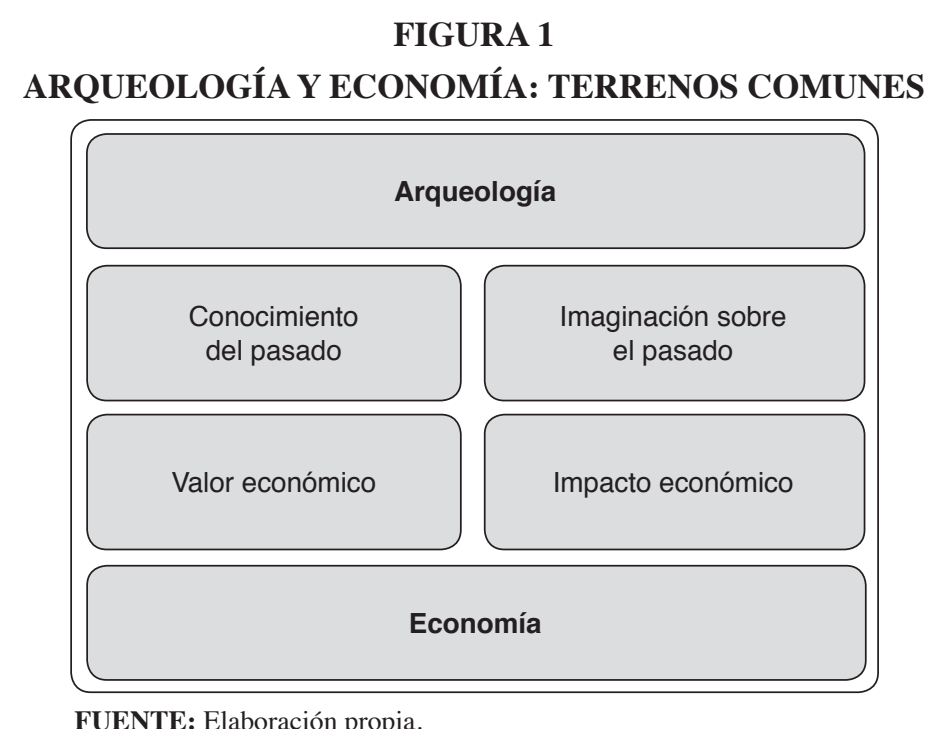

\section{Caracterización del patrimonio arqueológico como bien económico}

Partiendo de la tríada clásica de prototipos de bienes culturales dentro de la economía de la cultura, a saber, artes escénicas, patrimonio cultural e industrias culturales (Throsby, 2001; Towse, 2014), parece obvio entender que el patrimonio arqueológico pertenece al segundo tipo, es decir, como parte integrante de patrimonio histórico. En efecto, no se trata de un producto efímero que se agota en el mismo momento de la ejecución, como un concierto o una obra de teatro, sino que consiste en un recurso físico, perenne, queda para siempre, porque está desde siempre. Tiene además una particularidad por la que se hace diferenciar del resto de bienes del patrimonio histórico, como los monumentos, las artes plásticas, etc., que ya están construidos y son visibles, pues el patrimonio arqueológico, al menos la parte que comprende al trabajo de las excavaciones, está sometido a condiciones de incertidumbre e información imperfecta, ya que no se conocen sus características ni su alcance hasta que no se descubre, no se analiza y se interpreta. La composición de los artefactos del patrimonio arqueológico también es diferente, pues no se ciñe a meros objetos artísticos, sino que abarca todo tipo de materiales que constituyen un legado de lo encontrado o buscado, desde huesos humanos, cerámica, útiles y herramientas, restos de construcción urbana, etc., y cuyo valor se fundamenta en el grado de representatividad, antigüedad y estado de conservación. Por último, parece obvio también que no se trata de un elemento reproducible como los productos de las industrias culturales, sino que desde los tesoros más recónditos de la civilización egipcia, hasta las vasijas más humildes encontradas en un yacimiento vacceo de la meseta castellana, todos son artefactos únicos, singulares y no reproducibles (Burtenshaw, 2014; Zubrow, 2016). 
Sin embargo, y como hemos adelantado en la sección anterior, el patrimonio arqueológico es fuente de inspiración de nuevos productos y servicios en al ámbito de las industrias culturales y creativas, así como recibe usos complementarios de artes escénicas y musicales, o se integra como dotación renovada o modernas prestaciones culturales con nuevas funciones en la ordenación urbana. A ello debemos añadir toda la oferta de bienes y servicios adicionales asociados al uso turístico derivado del prototipo arqueológico, que en el caso de los grandes iconos (como Pompeya, Egipto, o las civilizaciones prehispánicas en México o Perú) puede ser trascendental para la economía del lugar o del país, originando incluso problemas de congestión y externalidades negativas (Comer, 2014; Ercolano et al., 2018). Por tanto, la correspondencia de la arqueología como cultura acumulada con la cultura viva y la cultura reproducible es un hecho destacable, y demostrativo de que lo importante es poseer y mantener el recurso, que es el activo fundamental del que emana y con el que posteriormente se puede construir una oferta cultural complementaria, con efectos en términos de impacto económico inducido (Herrero, 2011).

Pues bien, el patrimonio arqueológico, en tanto que bien característico del patrimonio histórico, reúne la siguiente caracterización desde el punto de vista del análisis económico. Señalaremos, en síntesis, cinco rasgos fundamentales. En primer lugar, y como se ha recalcado, se trata de un recurso fijo, único, no renovable e irreproducible (Comer, 2014). Esto implica que se trata de un mercado de oferta rígida, la del recurso único, y la manera de canalizar el valor no se realiza a través de precios sino de rentas de explotación, es decir, las que recaba el productor o poseedor del recurso en régimen de monopolio exclusivo por su propia naturaleza. La rentabilidad, en este caso, depende del uso y alcance del recurso, porque lógicamente no tiene el mismo impacto un pequeño yacimiento o un centro de interpretación que, por ejemplo, las ruinas de Pompeya, o el conjunto arqueológico de Angkor en Camboya, aunque el régimen de organización de recursos responda básicamente al mismo planteamiento. No obstante, resulta interesante añadir que un recurso cultural o arqueológico, aunque único, puede ver relajado su carácter monopólico cuando se inserta en un distrito cultural, donde los atractivos turísticos y culturales pueden competir entre sí, la oferta es más sustitutiva y por lo tanto elástica, y los precios pueden de nuevo canalizar mejor la intensidad de preferencias y la asignación de valor.

En segundo lugar, el patrimonio arqueológico es un bien raíz, es decir, un recurso inherente al territorio donde se localiza o donde se depositan los materiales encontrados, como museos y centros de interpretación. En la medida en que no es habitual la cesión y venta de estos materiales una vez inventariados en los museos, aunque tengan un carácter de bien mueble y por tanto desapegados del territorio, podemos entender que el patrimonio arqueológico constituye una dotación inamovible e idiosincrática del lugar donde se ubica. No obstante, lo relevante de esta característica es que, como también hemos mencionado, lo importante es poseer y mantener el recurso cultural, y alentar la producción de una oferta cultural complementaria de bienes y servicios, que puede convertirse en fuente de actividad productiva y de desarrollo económico. El patrimonio cultural y arqueológico puede entenderse, 
entonces, como una dotación de capital, un activo que rinde rentas en forma de flujo de bienes y servicios derivados, y que puede depreciarse si no se cuida o acumularse si se mejora y se invierte (Throsby, 1999). De ahí que los proyectos arqueológicos no deban limitarse a rescatar y estudiar, sino también de promover con carácter imaginativo y sostenible una producción cultural y complementaria derivada del recurso (Burtenshaw, 2014).

En tercer lugar, y asociado a su carácter único e irrepetible, el patrimonio arqueológico está sometido a condiciones de sostenibilidad, en el sentido de que, si se agrede o se destruye, desaparece para siempre en tanto que bien único e irrepetible. Estas amenazas pueden venir por causa de conflictos, desastres naturales o problemas en el programa de preservación y mantenimiento (Zubrow, 2016). Sin embargo, debemos añadir un peligro indirecto, como puede ser la congestión de visitantes, especialmente en el caso de grandes iconos arqueológicos, o incluso en los de menor entidad pero no cuidan y regulan las maneras de acceso y explotación. En ambos casos pueden generarse pérdidas irrecuperables y, cuando menos, costes significativos de reposición y mantenimiento (Pérez-Juez, 2006), por no hablar de los procesos de gentrificación que puede aniquilar la esencia de autenticidad y banalizar el respaldo cultural del patrimonio arqueológico.

Por lo que se refiere a las cuestiones de demanda, puede comprobarse que en el consumo de patrimonio arqueológico no se requiere un bien en particular, sino los componentes de valor que lleva incorporado o los servicios que puedan derivarse. El consumo se basa pues en características y prestaciones, que pueden ir, desde la emoción estética, hasta el valor cognitivo y de formación, el valor social como seña de identidad. También puede computarse el valor económico inducido de los productos derivados, es decir, la venta de entradas, catálogos, derechos de imagen, etc., así como los servicios de ocio y turismo que se relacionan con la visita. En esta interpretación resulta apropiado precisar que la mayor parte de los bienes culturales ofrecen siempre dos tipos de características: unas ex ante, relacionadas con las expectativas del bien deseado, y otras ex post, que juzgan la mercancía una vez realizado el consumo. En las primeras, las políticas de «labelización» del patrimonio cultural arqueológico pueden cumplir una función esencial en la formación de las preferencias, pero también en la probabilidad de congestión y maduración precipitada del recurso (Cuccia et al., 2016).

Por último y en relación a las condiciones de provisión del patrimonio arqueológico, cabe decir que la mayor parte de los sitios arqueológicos establecidos y las instalaciones expositivas derivadas tienen el carácter de bienes públicos o semipúblicos, es decir, cumplen con las condiciones de no rivalidad y no exclusividad una vez provistos. Con excepción de los casos extremos de congestión de demanda, el coste marginal de atender un visitante adicional es próximo a cero, y por tanto no hay precios relevantes que canalicen la provisión óptima de estos recursos en una economía de mercado. En consecuencia, en caso de estar a cargo del Estado, el precio sombra debería ser asumido íntegramente, o repercutido en la medida de lo posible, y si se cree conveniente, en las tarifas de visita. En este ámbito, existe también una 
opinión muy sugerente, cada vez más extendida, de considerar el patrimonio cultural y en particular el patrimonio arqueológico bajo la noción de bienes públicos globales (Benhamou, 2012; Comer, 2014), en el sentido de que pertenecen al disfrute colectivo mundial, propenden externalidades positivas de amplia gama (conocimiento, identidad, impacto económico, etc.) y la responsabilidad de su provisión y preservación desborda las fronteras y atañe a políticas de cooperación internacional. Lógicamente, esta calificación puede ser apropiada para los grandes conjuntos patrimoniales y arqueológicos de renombre mundial, pero queda diluida para la mayor parte del trabajo arqueológico habitual.

Más oportuna puede ser, en este sentido, la consideración de parte del legado arqueológico, sobre todo aquellos yacimientos por descubrir o en estudio de excavación, como bienes o recursos de propiedad común, es decir, aquellos que poseen las condiciones de rivalidad pero no exclusividad (Comer, 2004). En el extremo ocurriría como los bancos de pesca en alta mar, donde no existen barreras ni delimitaciones de propiedad que impidan la explotación particular, y cada pescador rivaliza en las capturas, sin incentivos para controlar su actividad, si no dispone de mecanismos de garantía cooperativa. Este argumento es lo que explica determinadas situaciones de pillaje y esquilmación de determinados yacimientos arqueológicos en situación de desamparo institucional o ausencia de regulación aclaratoria ${ }^{5}$; pero también la tendencia a desatenderlos o encubrirlos cuando los restos arqueológicos no tienen suficiente acreditación social. Por estas razones se suele asignar al Estado la función de suministro y atención adecuada del patrimonio cultural y particularmente del patrimonio arqueológico y evitar, así, los fallos de mercado debidos a la falta de delimitación de los derechos de propiedad y ausencia de mecanismos sociales de cooperación (Gestrich, 2011; Klamer, 2014). Pero esto puede generar también a medio plazo otros problemas relativos a la pérdida de reconocimiento social y fallos del Estado, como ahora veremos.

\section{Valor e impacto económico del patrimonio arqueológico}

Una de las cuestiones cruciales y más difíciles de resolver en economía de la cultura es la asignación de valor al patrimonio cultural y, por tanto, al patrimonio arqueológico, dada su especificidad, como hemos visto. Las razones de esta dificultad radican en que, a diferencia del resto de bienes y servicios de una economía, en los bienes culturales podemos distinguir dos acepciones de valor, ineludibles e inseparables, cual dos caras de una misma moneda: valor cultural y valor económico (Throsby, 2001). Para algunos esta disquisición es de por sí una provocación, porque piensan que la arqueología solo tiene valor cultural. Sin embargo, cuando

${ }^{5}$ Ver también el caso Odyssey, donde el Estado español ha debido de recurrir a un proceso jurídico de reclamación internacional para recuperar el tesoro de la fragata Mercedes, hundida en aguas cercanas al Algarve en 1804. 
indagamos por el valor económico, es que nos estamos preguntando, por ejemplo, por qué ha existido una campaña mundial, incluso expresada en crowfunding, para evitar los desastres de Palmira o los Budas de Bamiyan; o por el contrario, por qué se prefiere datar y ocultar (si no a veces destruir) restos arqueológicos cuando se construye un parking subterráneo; y, por último, por qué los yacimientos de Pompeya o Machu-Picchu constituyen iconos turísticos mundiales, con necesidad imperiosa de regulación del flujo turístico. En los tres casos se pone de manifiesto un problema de reconocimiento social del valor cultural del patrimonio arqueológico que, a su vez, tiene distintas implicaciones económicas, valoradas en términos del coste de oportunidad del legado cultural y su preservación.

Nos encontramos, no obstante, con un problema añadido, pues ambos valores, valor cultural y valor económico, tienen problemas para su revelación auténtica, el primero, porque no existen reglas uniformes que aseguren la consistencia en las elecciones individuales y se dirime, por tanto, mediante sanciones colectivas ${ }^{6}$; y el segundo, porque los bienes culturales y arqueológicos, como hemos visto, tienen problemas de definición y delimitación de las posibilidades de apropiabilidad, y por tanto el mercado y los precios no son eficientes en la revelación del valor (Klamer, 2014). Sin embargo, cuando ambos valores, cultural y económico, se reconocen y se asignan, bien como resolución colectiva o como voluntad de aprecio individual, provocan la aparición de dos nuevos estratos de valor, como si fueran dos nuevas ventanas derivadas, que implican, por un lado, la sanción social del significado cultural del prototipo arqueológico, y por otro, la aparición de mercados específicos y flujos económicos derivados de su valoración individual y social.

Por lo tanto y a nuestro juicio, en la configuración del valor del patrimonio arqueológico pueden distinguirse cuatro componentes, tal y como aparecen en la Figura 2 adjunta y que pasamos a explicar. En el primer cuadrante tenemos el «valor cultural». Se refiere al valor artístico, histórico, estético, científico y simbólico del patrimonio arqueológico. Su rango en la escala de valor depende de características relativamente objetivas como son su estado de conservación, grado de representatividad, rareza del artefacto, autenticidad, etc. Pero todas estas características son acreditadas por expertos, curadores y los propios arqueólogos, y por tanto sancionadas de forma colectiva en base al poder de delegación que se les otorga asociado a criterios sociales de mantenimiento y preservación. La razón se justifica porque es imposible en el terreno individual construir unas reglas de valor cultural válidas y estables en el tiempo y en el espacio para todas las personas, ya que depende, en esencia, tanto de las gustos particulares, como de los gustos acumulados en términos de experiencias previas de consumo, así como del nivel formativo de los individuos (Throsby, 2001; Herrero, 2001).

${ }^{6}$ Esta es la razón por la que la mayor parte de la regulación sobre arqueología se basa, en esencia, en la sanción potestativa del Estado a la protección y preservación del patrimonio arqueológico. 


\section{FIGURA 2}

\section{COMPONENTES DE VALOR DEL PATRIMONIO ARQUEOLÓGICO}

\section{Los valores del patrimonio cultural/arqueología}

\section{Valor cultural}

- Valor estético, histórico, carácter representativo, estado de conservación, autenticidad, rareza.

- Decisiones colectivas basadas en curadurías y expertos.

\section{Valor económico}

- Intensidad de preferencias, disposición a pagar, voluntad de aprecio. Curva de demanda.

- Valor del coste de oportunidad.

- Dificultad del mercado/precios como canalización del valor.
Valor social

- Identidad, simbolismo, branding nacional.

- Educación, formación, valor científico.

- Cohesión social, vinculación colectiva.

Impacto económico

- Flujos económicos en términos de empleo, gasto y actividad.

- Gastos directos, indirectos e inducidos.

- Valor de mercado: coleccionismo, subastas (expoliación).

\section{Necesidad de reconocimiento}

FUENTE: Elaboración propia.

En el segundo cuadrante y en la misma línea vertical de valores básicos, tenemos el denominado «valor económico». No nos estamos preguntando aquí por el volumen de gastos necesarios para una intervención arqueológica o para el mantenimiento de un legado, sino por la valoración intrínseca que atribuyen los individuos a estos bienes y que podría expresarse en una escala comprensible como el dinero, es decir los precios. Nos preguntamos, por tanto, por la voluntad de aprecio, por la intensidad de preferencias de las personas por el patrimonio cultural y el legado arqueológico, descubierto o por descubrir, y que lógicamente está íntimamente ligado a la acepción anterior, el valor cultural. Lo que ocurre es que no es fácil que se manifieste en forma de precios, como el resto de bienes de una economía. Además, existe la complicación adicional de que puede distinguirse no solo el valor de uso directo, sino también el valor de uso pasivo, es decir, aquel que se está dispuesto a sufragar aunque no se consuma directamente el bien, sino que se asigne valor a la opción de consumirlo en otro momento, la posibilidad de mantenerlo para futuras generaciones o simplemente porque se desea que exista. El análisis económico ha resuelto de manera más o menos convincente distintas técnicas en este sentido para estimar estos componentes de valor en forma de lo que se denomina disposición a pagar (Herrero, 2001), que 
no es más que una forma de asignar precios revelados o declarados para construir la verdadera finalidad, que es la curva de demanda del bien cultural.

En el tercer cuadrante y paralelo al valor cultural tenemos el «valor social». Cuando el patrimonio arqueológico es reconocido y acreditado en su valor cultural, se desencadenan unos efectos que trascienden al ámbito social y que contribuyen a reforzar la identidad de una sociedad y su sentido de pertenencia, pero también la marca de competitividad del enclave donde se sitúan, bien sea una ciudad, una región o un país en su conjunto ${ }^{7}$. Depende si el recurso arqueológico tiene la capacidad de convertirse también en icono cultural y turístico, en cuyo caso el nivel de reconocimiento repercute también en impacto económico, como ahora veremos. Sin embargo, debemos añadir en este punto, que el valor social del patrimonio arqueológico no solo toma cuerpo en forma de vinculación colectiva o reputación de una nueva imagen, sino que también propende en términos de formación, ya que la arqueología en sí misma es un recurso educativo y puede ser también un factor de desarrollo científico.

Por último, en el cuarto cuadrante se reseña el valor de «impacto económico». Se trata de un vector de valor real de mercado, pues concierne a la estimación de los flujos económicos involucrados en el uso y explotación del patrimonio arqueológico en términos de renta, empleo y actividad productiva. Comprende, en consecuencia, tanto los gastos de ejecución y mantenimiento de las actividades arqueológicas en todo su sentido (estudio, exploración, exhibición, etc.), como del flujo económico asociado a la demanda de estos recursos, donde el turismo es preponderante. Resulta obvio que estos valores resultan también de un proceso de reconocimiento anterior, tanto en el rango cultural del legado patrimonial, como en el significado y aprecio individual que puede alcanzar. Por esta razón, en este componente no solo distinguimos el impacto económico derivado, sino también la posibilidad de originar mercados específicos asociados al coleccionismo público o privado de artefactos arqueológicos. Estos mercados pueden concretarse en formas regladas como casas de subastas, ferias de anticuarios, comercio regular, pero también en operaciones fraudulentas fruto de expoliaciones o comercio furtivo (Zubrow, 2016).

La utilidad de la Figura 2 no se ciñe tan solo a la delimitación de los cuatro componentes de valor del patrimonio arqueológico, sino que resulta también interesante interpretarla en un doble sentido argumental ${ }^{8}$. Primero, como lectura horizontal en dos líneas paralelas, es decir, el valor cultural y el valor económico, que mutan a través del filtro de reconocimiento en valor social e impacto económico, respectivamente. Por otra parte, y bajo una lectura vertical, encontraríamos en primer lugar y a la izquierda de la figura, lo que constituyen decisiones de bienestar, es decir, las estimaciones de aprecio y significado del patrimonio arqueológico y que nos justifican

7 Pensemos, por ejemplo, en una escala creciente, el carácter emblemático de algunos monumentos aislados para ciudades como Segovia en España con su acueducto romano, o los restos de civilizaciones prehispánicas, auténtica riqueza y seña de identidad para México como país, o el efecto icónico a nivel mundial del conjunto arqueológico de Stonhenge en Reino Unido.

${ }^{8}$ Ver también una estructura explicativa parecida en Klamer (2014). 
las decisiones de preservarlo, estudiarlo y rescatarlo. En segundo lugar y a la derecha tendríamos el cómputo de los efectos tangibles e intangibles que se deducen del anterior reconocimiento y valoración, es decir, impacto económico y valor social.

En este juego explicativo, la economía no entra con facilidad en las interpretaciones de valor cultural y valor social, aunque los considera. Pero sí se arriesga en la estimación del valor económico y el impacto de flujos derivados del patrimonio cultural y arqueológico, que en el fondo deben ser y son un espejo del alcance de los dos primeros valores. Para ello se han desarrollado una serie de técnicas que tienen ya una gran extensión en aplicaciones en el ámbito del sector cultural, pero aún no tantas en el área específica de la arqueología. Una síntesis de los mismos aparece recopilada en la Tabla 1, que constituye una compilación de lo que, desde nuestro conocimiento, se ha venido publicando sobre ejercicios de valoración y análisis del patrimonio arqueológico desde el punto de vista económico9 ${ }^{9}$ Dividimos estos trabajos en dos grupos: ensayos de valoración económica y aplicaciones de impacto económico y turístico de legados y artefactos arqueológicos.

Por lo que se refiere al primer grupo de trabajos, es decir, los métodos de estimación del valor económico intrínseco del patrimonio a través de la disposición a pagar por parte de los individuos, algunos modelos lo realizan de manera indirecta a través de mercados paralelos, como el coste de transporte (método del coste del viaje) o el mercado inmobiliario (método de precios hedónicos). Las preferencias en estos casos son reveladas y se limitan a deducir el valor de uso directo del bien cultural objeto de estudio. En este marco, podemos destacar los estudios de valoración de patrimonio arqueológico, por ejemplo, de la Cueva del Soplao en Cantabria, España (Pérez-Álvarez, 2016) o el templo de Poseidón en Grecia (Tourkolias et al., 2015) a través de la técnica del coste del viaje; o la valoración de yacimientos arqueológicos en el área de Dublín (Moro et al., 2013) mediante el método de precios hedónicos. De forma paralela se han desarrollado otras técnicas que tratan de obtener el valor económico del bien cultural a través de la creación de un mercado directo, contingente, en el que la oferta cultural es planteada por el encuestador y las preferencias y la disposición a pagar son declarados por los entrevistados. En este marco se han desarrollado técnicas profusamente utilizadas, como el método de valoración contingente y el método de experimentos de elección. Con el primer modelo, la lista de aplicaciones es amplia y se han evaluado determinados yacimientos arqueológicos en México (Beltrán y Rojas, 1996) y la evaluación de cambios en el entorno del conjunto arqueológico de Stonehenge (Maddison y Mourato, 2001); mientras que con el segundo método encontramos aplicaciones para el Muro de Adriano en el Reino Unido (Kinghorn y Willis, 2008) y algunos yacimientos de la edad de piedra en Dinamarca (Lundhede et al., 2013).

\footnotetext{
${ }^{9}$ Se ha realizado una selección a partir de la base de datos Web of Science, limitando la consulta a artículos publicados en revistas científicas que presenten un propósito de estudio de algún legado o artefacto arqueológico desde el punto de vista económico y un perfil metodológico afín.
} 


\section{TABLA 1}

COMPILACIÓN DE ESTUDIOS ECONÓMICOS DEL PATRIMONIO CULTURAL ARQUEOLÓGICO

\begin{tabular}{|c|c|c|c|}
\hline Referencia & $\begin{array}{l}\text { Bien o yacimiento } \\
\text { arqueológico }\end{array}$ & Objetivos de estudio & Metodología \\
\hline \multicolumn{4}{|c|}{ Estudios de valoración económica } \\
\hline $\begin{array}{l}\text { Beltrán y Rojas } \\
\text { (1996) }\end{array}$ & $\begin{array}{l}\text { Yacimientos arqueológicos, } \\
\text { México }\end{array}$ & $\begin{array}{l}\text { Estimación de la disposi- } \\
\text { ción a paga por el consumo } \\
\text { y preservación de sitios ar- } \\
\text { queológicos }\end{array}$ & Valoración contingente \\
\hline $\begin{array}{l}\text { Lundhede } \text { et al. } \\
\text { (2013) }\end{array}$ & $\begin{array}{l}\text { Yacimientos edad de piedra, } \\
\text { Dinamarca }\end{array}$ & $\begin{array}{l}\text { Estimación de valor de uso } \\
\text { pasivo y preservación del } \\
\text { patrimonio arqueológico }\end{array}$ & Experimentos de elección \\
\hline $\begin{array}{l}\text { Kinghorn y Willis } \\
(2008)\end{array}$ & $\begin{array}{l}\text { Muro de Adriano, Gran Bre- } \\
\text { taña }\end{array}$ & $\begin{array}{l}\text { Valoración de componentes } \\
\text { de valor y alternativas de } \\
\text { gestión del sitio arqueoló- } \\
\text { gico }\end{array}$ & Experimentos de elección \\
\hline $\begin{array}{l}\text { Maddisson y Mou- } \\
\text { rato (2002) }\end{array}$ & $\begin{array}{l}\text { Sitio arqueológico Stone- } \\
\text { henge, Reino Unido }\end{array}$ & $\begin{array}{l}\text { Valoración de planes de ac- } \\
\text { ceso a Stonehenge }\end{array}$ & Valoración contingente \\
\hline Moro et al. (2013) & $\begin{array}{l}\text { Yacimientos arqueológicos } \\
\text { y patrimonio histórico, Du- } \\
\text { blín, Irlanda }\end{array}$ & $\begin{array}{l}\text { Estimación de externalida- } \\
\text { des y valor del patrimonio } \\
\text { cultural y arqueológico }\end{array}$ & Precios hedónicos \\
\hline $\begin{array}{l}\text { Pérez Álvarez et al. } \\
\text { (2019) }\end{array}$ & $\begin{array}{l}\text { Cueva del Soplao, Canta- } \\
\text { bria, España }\end{array}$ & $\begin{array}{l}\text { Valoración de uso turísti- } \\
\text { co de conjunto patrimonial } \\
\text { minero }\end{array}$ & Coste del viaje \\
\hline $\begin{array}{l}\text { Tourkolias et al. } \\
\text { (2015) }\end{array}$ & $\begin{array}{l}\text { Templo de Poseidón, Sou- } \\
\text { nio, Grecia }\end{array}$ & $\begin{array}{l}\text { Estimación del valor econó- } \\
\text { mico asignado por visitantes }\end{array}$ & Coste del viaje \\
\hline \multicolumn{4}{|c|}{ Estudios de impacto económico y turístico } \\
\hline $\begin{array}{l}\text { Báez y Herrero } \\
(2012)\end{array}$ & $\begin{array}{l}\text { Conjunto histórico urbano } \\
\text { de Valdivia, Chile }\end{array}$ & $\begin{array}{l}\text { Valor de uso directo y pa- } \\
\text { sivo del conjunto y evalua- } \\
\text { ción de plan de recuperación }\end{array}$ & $\begin{array}{l}\text { Valoración contingente y } \\
\text { análisis coste beneficio }\end{array}$ \\
\hline Brida et al. (2012) & $\begin{array}{l}\text { Museo arqueológico del } \\
\text { Tirol del Sur, Museo Ötzi, } \\
\text { Bolzano, Italia }\end{array}$ & $\begin{array}{l}\text { Análisis de motivación y } \\
\text { lealtad turística }\end{array}$ & $\begin{array}{l}\text { Coste del viaje y regresión } \\
\text { de Poisson }\end{array}$ \\
\hline Burch et al. (2019) & $\begin{array}{l}\text { Restos arqueológicos de la } \\
\text { Ciudadela de Rosas, España }\end{array}$ & $\begin{array}{l}\text { Valoración de precio de en- } \\
\text { trada y estrategias de uso }\end{array}$ & Coste del viaje \\
\hline $\begin{array}{l}\text { Ercolano et al. } \\
\text { (2018) }\end{array}$ & $\begin{array}{l}\text { Área arqueológica del Vesu- } \\
\text { bio, Italia }\end{array}$ & $\begin{array}{l}\text { Análisis de motivación tu- } \\
\text { rística y competencia entre } \\
\text { sitios arqueológicos }\end{array}$ & $\begin{array}{l}\text { Análisis de corresponden- } \\
\text { cias y regresión logística }\end{array}$ \\
\hline $\begin{array}{l}\text { Martín-Ruiz et al. } \\
\text { (2010) }\end{array}$ & $\begin{array}{l}\text { Área arqueológica de Santi- } \\
\text { ponce, España }\end{array}$ & $\begin{array}{l}\text { Construcción de índices de } \\
\text { evaluación de visitas turís- } \\
\text { ticas }\end{array}$ & $\begin{array}{l}\text { Modelos de ecuaciones es- } \\
\text { tructurales }\end{array}$ \\
\hline $\begin{array}{l}\text { Murillo et al. } \\
\text { (2008) }\end{array}$ & $\begin{array}{l}\text { Conjunto de La Alhambra y } \\
\text { Generalife }\end{array}$ & $\begin{array}{l}\text { Estimación del impacto } \\
\text { económico del conjunto de } \\
\text { patrimonio cultural }\end{array}$ & $\begin{array}{l}\text { Modelos de impacto econó- } \\
\text { mico }\end{array}$ \\
\hline $\begin{array}{l}\text { Tuan y Navrud } \\
\text { (2008) }\end{array}$ & $\begin{array}{l}\text { Santuario arqueológico de } \\
\text { My Son, Vietnam }\end{array}$ & $\begin{array}{l}\text { Medición del beneficio so- } \\
\text { cial y económico y progra- } \\
\text { ma de preservación }\end{array}$ & $\begin{array}{l}\text { Valoración contingente y } \\
\text { análisis coste beneficio }\end{array}$ \\
\hline
\end{tabular}

FUENTE: Elaboración propia. 
En la otra vertiente, la de los estudios de impacto económico y turístico, podemos distinguir, a su vez, tres tipos de análisis. Primero, aquellos que utilizando resultados de valoración económica, plantean la evaluación del rendimiento social de un plan de recuperación o preservación del patrimonio arqueológico, como son los casos del conjunto arqueológico de My Son en Vietnam (Tuan y Navrud, 2008) o el patrimonio cultural urbano de Valdivia en Chile (Báez y Herrero, 2012). Burch et al., (2019) también plantean para el área arqueológica de la Ciudadela de Rosas en España distintas estrategias de uso a medio y largo plazo fundamentadas en una estimación de precio de entrada o acceso gratuito. En segundo lugar, tenemos específicamente los trabajos orientados a la evaluación de las experiencias turísticas o el análisis de motivación y lealtad del visitante, temas que tienen mayor profusión en la literatura de economía del turismo. Aquí resaltamos los trabajos de Martín-Ruiz et al. (2010) sobre el conjunto arqueológico de Santiponce (España), Brida et al. (2012) respecto del Museo arqueológico de Bolzano (Museo Ötzi), Italia, y Ercolano et al. (2018) con un análisis de motivación turística y estudio interesante de competencia entre sitios en el área arqueológica del Vesubio, Italia. Por último, tendríamos los estudios de impacto económico en sentido estricto, es decir, los que tratan de computar los efectos directos, indirectos e inducidos de un conjunto patrimonial, donde resaltamos, por ejemplo, el trabajo de Murillo et al. (2008) sobre La Alhambra y el Generalife.

\section{Conclusión}

En este ensayo hemos querido demostrar la capacidad explicativa del análisis económico para entender y valorar las decisiones de asignación de recursos en el ámbito de la arqueología. Se ha llegado a caracterizar el patrimonio arqueológico desde el punto de vista económico, precisando la especificidad de este prototipo dentro del espectro del patrimonio cultural. Es importante destacar que el patrimonio arqueológico puede entenderse como un capital, como un activo inmanente al territorio o al enclave donde se ubica, mientras que la oferta cultural relacionada es circunstancial, cuya eficacia y sostenibilidad depende del grado de imaginación y criterio en su configuración, con el fin de evitar problemas de degradación o pérdida de identidad del elemento patrimonial. Se ha profundizado también en los componentes de valor del patrimonio arqueológico, distinguiendo entre valor cultural, valor económico, valor social e impacto económico. Los dos primeros son intrínsecos al bien arqueológico, mientras que los dos segundos dependen del proceso de reconocimiento individual y colectivo de una sociedad. La economía puede brindar técnicas pertinentes que traten de estimar de manera rigurosa el valor económico y el impacto en términos de flujos del patrimonio cultural y arqueológico. Distintas aplicaciones en este sentido han sido presentadas a modo de referencia comparada. 


\section{Referencias bibliográficas}

Báez, A., \& Herrero, H. (2012). Using contingent valuation and cost-benefit analysis to design a policy for restoring cultural heritage. Journal of Cultural Heritage, 10(2), 235-245.

Beltrán, E., \& Rojas, M. (1996). Diversified funding methods in Mexican archaeology. Annals of Tourism Research, 23(2), 463-478.

Benhamou, F. (2012). Économie du patrimoine culturel. París: Éditions La Découverte.

Brida, J.G., Meleddu, M., \& Pulina, M. (2012). Understanding Urban Tourism Attractiveness: The Case of the Archaeological Ötzi Museum in Bolzano. Journal of Travel Research, 51(6), 730-741.

Buchanan, A. (2005). Industrial Archaeology: Past, Present and Prospective. Industrial Archaeology Review, 27(1), 19-21.

Burch, J., Rigall, R., Saló, A., \& Alcalde, G. (2019). Free or paid: the uses of Roses Citadel (Catalonia). Journal of Cultural Heritage Management and Sustainable Development, 9(1), 111-120.

Burtehnshaw, P. (2014). Mind the Gap: Cultural and Economic Values in Archaeology. Public Archaeology, 13(1-3), 48-58.

Comer, D. (2014). Threats to the Archaeological Heritage in the Laissez-Faire World of Tourism: The Need for Global Standards as a Global Public Good. Public Archaeology, 13(1-3), 123-134.

Cuccia, T., Guccio, C., \& Rizzo, I. (2016). The effects of UNESCO World Heritage List inscription on tourism destinations performance in Italian regions. Economic Modelling, 53, 494-508.

Demas, M., \& Agnew, N. (2013). Conservation and sustainable development of archaeological sites. En I. Rizzo y A. Mignosa, Handbook on the Economics of Cultural Heritage (pp. 326-343). Chentelham: Edward Elgar.

Ercolano, S., Gaeta, G., \& Parenti, B. (2018). Pompeii dilemma: A motivation-based analysis of tourists' preference for "superstar" archaeological attractors or less renowned archaeological sites in the Vesuvius area. International Journal of Tourism Research, 20, 345-354.

Fernández Díaz, M. (2016). Reflexiones sobre la aplicación de tecnologías al trabajo arqueológico y la divulgación científica del patrimonio. La Linde, 6, 64-78.

Gestrich, N. (2011). Putting a Price on the Past: The Ethics and Economics of Archaeology in the Marketplace. A Reply to What is Public Archaeology. Present Past, 3(2), 80-82.

Gould, G., \& Burtehnshaw, P. (2014). Archaeology and Economic Development. Public Archaeology, 13(1-3), 3-9.

Herrero, L. C. (2001). Economía del patrimonio histórico. Información Comercial Española. Revista de Economía, 792, 151-168.

Herrero, L. C. (2011). La contribución de la cultura y las artes al desarrollo económico regional. Investigaciones Regionales, 19, 177-202.

Kinghorn, N., \& Willis, K. (2008). Valuing the components of an archaeological site: An application of Choice Experiment to Vindolanda, Hadrian's Wall. Journal of Cultural Heritage, 9, 117-124.

Klamer, A. (2014). The Values of Archaeological and Heritage Sites. Public Archaeology, 13(1-3), 59-70. 
Lundhede, T., Bille, T., \& Haslerd, B. (2013). Exploring preferences and non-use values for hidden archaeological artefacts: a case from Denmark. International Journal of Cultural Policy, 19(4), 501-530.

Maddison, D., \& Mourato, S. (2001). Valuing different road options for Stonehenge. Journal of Conservation and Management of Archaeological Sites, 4.

Martín-Ruiz, D., Castellanos-Verdugo,M.,\& Oviedo-García, M.(2010). A visitors' evaluation index for a visit to an archaeological site. Tourism Management, 31(5), 590-596.

Moro, M., Mayor, K., Lyons, S., \& Tol, R. S. (2013). Does the housing market reflect cultural heritage? A case study of Greater Dublin. Environment and Planning A: Economy and Space, 45(12), 2884-2903.

Murillo, J., Romaní, J., \& Suriñach, J. (2008). The impact of heritage tourism on an urban economy: the case of Granada and the Alhambra. Tourism Economics, 14(2), 361-376.

Paardekooper, R. (2012). The Value of an Archaeological Open-Air Museum is in its Use: Understanding Archaeological Open-Air Museums and their Visitors. Leiden: Sidestone Press.

Pérez-Álvarez, R., Torres-Ortega, S., Díaz-Simal, P., Husillos-Rodríguez, R., \& De LuisRuiz, J. (2016). Economic Valuation of Mining Heritage from a Recreational Approach: Application to the Case of El Soplao Cave in Spain. Sustainability, 8(2), 3-15.

Pérez-Juez Gil, A. (2006). Gestión del Patrimonio Arqueológico. Barcelona: Ariel.

Ross, D., Saxena, G., Correia, F., \& Deutz, P. (2017). Archaeological tourism: A creative approach. Annals of Tourism Research, 67, 37-47.

Throsby, D. (1999). Cultural Capital. Journal of Cultural Economics, 23(1-2), 3-12.

Throsby, D. (2001). Economics and Culture. Cambridge: Cambridge University Press.

Towse, R. (2014). Advanced Introduction to Cultural Economics. Elgar Advanced Introductions.

Tourkolias, C., Skiada, T., Mirasgedis, S., \& Kiakoulaki, D. (2015). Application of the travel cost method for the valuation of the Poseidon temple in Sounio, Greece. Journal of Cultural Heritage, 16, 567-574.

Tuan, T. H., \& Navrud, S. (2008). Capturing the benefits of preserving cultural heritage. Journal of Cultural Heritage, 9(3), 326-337.

Vecco, M. (2010). A definition of cultural heritage: From the tangible to the intangible. Journal of Cultural Heritage, 11, 321-324.

Weigel, W. (2006). Some economics of archaeology. En $14^{\text {th }}$ International Conference on Cultural Economics of the Association for Cultural Economics International, Viena (pp. 6-9).

Zubrow, E. B. W. (2016). Archaeological Cultural Heritage: A Consideration of Loss by Smuggling, Conflict or War. En I. Rizzo y R. Towse (Eds.), The Artful Economist. A New Look at Cultural Economics (pp. 215-226). Springer. 